\begin{tabular}{|c|l|}
\hline Title & Gait posture estimation using wearable acceleration and gyro sensors \\
\hline Author(s) & Takeda, Ryo; Tadano, Shigeru; Natorigawa, A kiko; Todoh, Masahiro; Y oshinari, Satoshi \\
\hline Citation & $\begin{array}{l}\text { Journal of Biomechanics, 42(15), 2486-2494 } \\
\text { https:/doi.org/40.1016/.jbiomech.2009.07.016 }\end{array}$ \\
\hline Issue Date & 2009-11-13 \\
\hline Doc URL & http://hdl.handle.net/2115/42483 \\
\hline Type & article (author version) \\
\hline File Information & JB42-15_2486-2494.pdf \\
\hline
\end{tabular}

Instructions for use 


\title{
Gait Posture Estimation using Wearable Acceleration and
}

\section{Gyro Sensors}

\author{
Ryo Takeda ${ }^{\text {a }}$, Shigeru Tadano ${ }^{a^{*}}$, Akiko Natorigawa ${ }^{a}$, Masahiro Todoh ${ }^{a}$ \\ Satoshi Yoshinari $^{\mathrm{b}}$
}

a Division of Human Mechanical Systems and Design, Graduate School of Engineering, Hokkaido University, Sapporo, Japan

${ }^{\mathrm{b}}$ Human Engineering Section, Product Technology Department, Hokkaido Industrial Research Institute, Sapporo, Japan

* Corresponding Author:

Shigeru TADANO, PhD

Professor, Division of Human Mechanical Systems and Design, Graduate School of Engineering, Hokkaido University

N13 W8, Kita-ku, Sapporo 060-8628, Japan

Tel/Fax: +81-11-706-6405, E-mail: tadano@eng.hokudai.ac.jp

Keywords: Gait Analysis; Acceleration Sensor; Gyro Sensor

Word count: 2982 words (Introduction through Discussion)

Manuscript Type: Original Article 


\section{Abstract}

A method for gait analysis using wearable acceleration sensors and gyro sensors is proposed in this work. The volunteers wore sensor units that included a tri-axis acceleration sensor and three single axis gyro sensors. The angular velocity data measured by the gyro sensors were used to estimate the translational acceleration in the gait analysis. The translational acceleration was then subtracted from the acceleration sensor measurements to obtain the gravitational acceleration, giving the orientation of the lower limb segments. Segment orientation along with body measurements were used to obtain the positions of hip, knee, and ankle joints to create stick figure models of the volunteers. This method can measure the three dimensional positions of joint centers of the hip, knee, and ankle during movement. Experiments were carried out on the normal gait of three healthy volunteers. As a result, the flexion-extension (F-E) and the adduction-abduction (A-A) joint angles of the hips and the flexion-extension (F-E) joint angles of the knees were calculated and compared with a camera motion capture system. The correlation coefficients were above 0.88 for the hip F-E, higher than 0.721 for the hip A-A, better than 0.924 for the knee F-E. A moving stick figure model of each volunteer was created to visually confirm the walking posture. Further, the knee and ankle joint trajectories in the horizontal plane showed that the left and right legs were 
bilaterally symmetric.

$-3-$ 


\section{Introduction}

Gait analysis is a clinical tool for obtaining quantitive information of the gait of a person to diagnose walking disabilities. Common methods of gait analysis include using cameras to track the position of body-mounted reflective markers, from which information on joint and limb segment motion can be derived. However, such systems are large, expensive and complex. Therefore, measurements are usually restricted to indoor laboratories.

An alternative method for measuring human motion is by placing small acceleration sensors on the body (Morris, 1973). Such inertial sensors allow measurements to be made outside the laboratory environment (Veltink et al., 1996, Response A-1

Bouten et al., 1997, Bussmann et al., 1998, Foerster et al., 1999). In contrast to conventional camera systems, inertial sensor systems do not measure positions.

Therefore, many reports have proposed methods to calculate three dimensional positions.

A common method to estimate body segment orientation is by integrating angular velocity data measured by gyro sensors worn on body segments (Tong and Granat, 1999). However, here small errors in the angular velocity data accumulate with integration, resulting in errors in the body segment orientation calculations. To reduce 
the extent of the integration errors, signal filtering based on assumptions of the cyclic Response

properties of gait has been applied. However this has been restricted to the measurements of cyclic gait. Further, method is the use of neural networks to predict joint angles from acceleration and angular velocity data (Findlow et al., 2008). Here high correlations were reported with the camera analysis, however the creation of such a prediction system requires much training data for the neural network before accurate predictions can be made. Other methods have used sophisticated Kalman filters to eliminate errors included in the sensor data to provide accurate three dimensional segment calculations (Luinge and Veltink. 2005). In addition, a combination of acceleration, gyro, and magnetic sensors were used to increase the accuracy (Zhu and Zhou, 2004, Roetenberg et al., 2007). However, reports showed that there were three dimensional orientation accuracy errors when compared with the camera analysis even in static states (Brodie et al., 2008). To avoid such errors, recalibration of the sensors had to be conducted regularly. Further, magnetic sensors are affected by ferrous compounds, and careful attention must be given to the magnetic surroundings and even the storage of the sensors.

Theoretically, it is possible to estimate the orientation of segments by the gravitational acceleration measured by acceleration sensors. However, in dynamic states 
such as the gait, a translational acceleration component will become included. The authors (Takeda et al., 2009) have used the cyclic patterns in acceleration data during gait to create an algorithm to obtain optimal gravitational acceleration patterns. Here, an optimal three dimensional representation for a person in the base coordinate system was reported, but there were differences in joint angles established with a camera based system. In addition, the method was only applicable to cyclic motion such as the gait.

Utilization of signal filters and optimization algorithms for acceleration and angular velocity data limited measurements to cyclic gait, and the use of magnetic sensors is not suitable for measurements in home environments. The work reported here proposes a method for gait analysis using only acceleration and gyro sensors that measure various kinds of gait in home environments. Here, the angular velocity was used to calculate the translational acceleration during the gait. The estimated translational acceleration was then subtracted from the measured acceleration data to obtain the gravitational acceleration. The gravitational acceleration provided the orientation angle of the segments and consequently the three dimensional posture of lower limb segments. To test the method, the gaits of three healthy volunteers were measured during walking on a flat floor. As a result, the hip flexion-extension (F-E), hip abduction-adduction (A-A) and knee flexion-extension (F-E) were estimated. The 
characteristic three-dimensional walking established by this method could be visualized in the form of a stick figure model moving in a base coordinate system. 


\section{Method}

\subsection{Sensor System}

The sensor system used in this investigation consisted of small wearable sensor units, each containing a data logger and a sensor head. The sensor head has a tri-axial acceleration sensor (H34C, Hitachi Metals, Ltd.) and three gyro sensors (ENC-03M, muRata Manufacturing Co., Ltd.), and one sensor unit can measure the acceleration and the angular velocity along three orthogonal axes simultaneously. The data logger can record the acceleration and angular velocity data for a maximum of 160 seconds at a sampling rate of $100 \mathrm{~Hz}$. One sensor unit weighs $136 \mathrm{~g}$, including battery (90 g), and the size is $50 \mathrm{~mm} \times 50 \mathrm{~mm} \times 15 \mathrm{~mm}$ for the data logger and $15 \mathrm{~mm} \times 15 \mathrm{~mm} \times 15 \mathrm{~mm}$ for the sensor head. All sensor units were checked on a mechanical turntable to establish the offset values for acceleration and angular velocity data, in addition to obtaining the inclination relationships of the measured values. These data were used for the initial zero offset of the sensors and for converting measured values to acceleration and angular velocity during the analysis. 


\subsection{Using acceleration sensors as inclination sensors}

Sensor units are placed on the lower limb segments of the volunteers as shown

in Fig. 1. Sensor units are placed on four body segments, on both thighs and both shanks (RT, LT, RS, and LS). In this report, the length and inclination of each segment was used to calculate the joint positions of both left and right hips, both knees, and both ankles (RH, LH, RK, LK, RA, and LA) during walking.

First, a tri-axial acceleration sensor is used as an inclination sensor, as it can measure the gravitational acceleration, and the output of an acceleration sensor $O_{i}$ can be expressed as

$$
O_{i}=a_{i}-g_{i} \quad(i=x, y, z)
$$

Here, $a_{i}$ is the translational acceleration and $g_{i}$ is the gravitational acceleration, both measured along the $i$ axis of the acceleration sensor. If the acceleration sensor is static the $a_{i}$ is 0 , meaning that the gravitational acceleration is the only output. Therefore, the angle of inclination for the three axes of an acceleration sensor against the gravitational acceleration direction can be expressed as

$$
\theta_{i}=\cos ^{-1} O_{i} / g
$$

and the gravitational acceleration as

$$
g=\sqrt{O_{x}^{2}+O_{y}^{2}+O_{z}^{2}}
$$




\subsection{Lower limb posture calculation}

The following vectors are used for calculating the hip joint and knee joint angles.

$$
\begin{aligned}
& \mathbf{g}=\mathbf{g}\left(g_{x}, g_{y}, g_{z}\right) \\
& \mathbf{a}_{\mathbf{B}}=\mathbf{a}_{\mathbf{B}}\left(a_{B x}, a_{B y}, a_{B z}\right) \\
& \mathbf{O}_{\mathbf{B}}=\mathbf{O}_{\mathbf{B}}\left(O_{B x}, O_{B y}, O_{B z}\right) \\
& \boldsymbol{\omega}_{\mathbf{B}}=\boldsymbol{\omega}_{\mathbf{B}}\left(\omega_{B x}, \omega_{B y}, \omega_{B z}\right) \\
& \mathbf{r}_{\mathbf{J B}}=\mathbf{r}_{\mathbf{J B}}\left(0,0,-r_{J B}\right)
\end{aligned}
$$

The terms used in these equations are detailed in Table 1, and a moving average of 15 data points was used to remove noise in the raw acceleration and angular velocity data.

\subsection{Measurements of hip joint angles}

The hip joint angle can be calculated with the inclination angle of the thigh segment, and the acceleration and angular velocity data for the thigh segment, LT and RT, are used to estimate the segment inclination.

Since gait is a dynamic state, $a_{i}$ in Eq. (1) must be determined before Eq. (2) can be used to calculate the angles of inclination for the $\mathrm{x}, \mathrm{y}$, and $\mathrm{z}$ axes of the sensor 
unit. A simple model for thigh and shank is shown in Fig. 2(a), here RH, RK, and RA are the centers of the joints for the hip, knee, and ankle of the right leg, and $S^{\mathrm{RT}}$ and $\mathrm{S}^{\mathrm{RS}}$ are the centers of the sensor units placed on the thigh and shank respectively. The calculations for the $\mathrm{S}^{\mathrm{RT}}$ can be divided into translational motion and rotational motion.

The movement of the hip joint angle is complex, and the thigh and shank was considered as a double pendulum with the center of the hip joint as the fulcrum. For simplification, it was assumed that the centripetal and tangential accelerations were dominant and that there was no translational acceleration, so translational acceleration becomes 0 , and only the rotational motion needs to be calculated. The rotational acceleration for RH can be expressed as

$$
\ddot{\mathbf{r}}_{\mathrm{HT}}=\dot{\boldsymbol{\omega}}_{\mathrm{T}} \times \mathbf{r}_{\mathrm{HT}}+\boldsymbol{\omega}_{\mathrm{T}} \times\left(\boldsymbol{\omega}_{\mathrm{T}} \times \mathbf{r}_{\mathrm{HT}}\right)
$$

with $\ddot{\mathbf{r}}_{\mathbf{H T}}$ the only acceleration, Eq. 1 is transformed into

$$
\mathbf{g}=\ddot{\mathbf{r}}_{\mathrm{HT}}-\mathbf{O}_{\mathrm{T}}
$$

and now the inclination of the thigh $\theta_{x}, \theta_{y}$, and $\theta_{z}$ can be calculated by Eq. (2).

In this work, Euler angles are adopted to convert segment inclination angles into hip joint angles, and the conversions used in Davis et al. (1991) was used. The Pitch angle will be considered the flexion and extension, the Roll angle the abduction and adduction, and the Yaw angle the inner and exterior rotation of the hip joint. The 
Yaw angle will not be considered in the calculations of this work, and the Pitch and Roll angles are obtained by the following equations

$$
\begin{aligned}
& \text { Pitch }=\theta_{x}-90^{\circ} \\
& \cos (\text { Pitch }) \times \sin (\text { Roll })=\cos \theta_{y}
\end{aligned}
$$




\subsection{Measurements of knee joint angles}

The acceleration and angular velocity data from the thigh segments, LT and RT, and the shanks, LS and RS, are used to estimate the knee F-E. The translational acceleration $\mathbf{a}_{\mathbf{T}}$, can be expressed using the following equations

$$
\begin{aligned}
& \mathbf{a}_{\mathrm{T}}=\mathbf{a}_{\mathrm{K}}+\ddot{\mathbf{r}}_{\mathrm{KT}} \\
& \ddot{\mathbf{r}}_{\mathrm{KT}}=\dot{\boldsymbol{\omega}}_{\mathrm{T}} \times \mathbf{r}_{\mathrm{KT}}+\omega_{\mathrm{T}} \times\left(\omega_{\mathrm{T}} \times \mathbf{r}_{\mathrm{KT}}\right)
\end{aligned}
$$

Here $\mathbf{a}_{\mathbf{K}}$ is the acceleration at a joint RK or LK, and the acceleration outputs of $\mathrm{S}^{\mathrm{RT}}$ or $\mathrm{S}^{\mathrm{LT}}$ can be expressed as

$$
\mathbf{O}_{\mathrm{T}}=\mathbf{a}_{\mathrm{T}}-\mathbf{g}
$$

If $\ddot{\mathbf{r}}_{\mathbf{K T}}$ is subtracted from both sides of Eq. (19) it be expanded as

$$
\mathbf{O}_{\mathrm{T}}=\left(\mathbf{a}_{\mathbf{H}}-\ddot{\mathbf{r}}_{\mathrm{KT}}\right)-\mathbf{g}=\mathbf{a}_{\mathrm{K}}-\mathbf{g}
$$

Since the $\mathbf{a}_{\mathbf{K}}-\mathbf{g}$ measured from $\mathrm{S}^{\mathrm{RT}}$ (or $\mathrm{S}^{\mathrm{LT}}$ ) and $\mathrm{S}^{\mathrm{RS}}$ (or $\mathrm{S}^{\mathrm{LS}}$ ) should be the same, the following equation would hold

$$
\mathbf{a}_{\mathrm{K}}-\mathbf{g}=\mathbf{O}_{\mathrm{T}}-\ddot{\mathbf{r}}_{\mathrm{KT}}=\mathbf{O}_{\mathrm{S}}-\ddot{\mathbf{r}}_{\mathrm{KS}}
$$

The method for obtaining knee joint angles is shown in Fig. 2(b1) and 2(b2). Here $\theta_{1}$ is the angle of inclination of $\mathbf{a}_{\mathbf{K}}-\mathbf{g}$ in relation to RT (or LT) segment and $\theta_{2}$ is the angle of inclination of $\mathbf{a}_{\mathbf{K}}-\mathbf{g}$ in relation to RS (or LS) segment. The values of $\theta_{1}$ and $\theta_{2}$ can be calculated by the following equations 


$$
\begin{aligned}
& \theta_{1}=\tan ^{-1} \frac{\left|\mathbf{O}_{\mathbf{T}}-\ddot{\mathbf{r}}_{\mathbf{K T}}\right|_{x}}{\left|\mathbf{O}_{\mathbf{T}}-\ddot{\mathbf{r}}_{\mathbf{K T}}\right|_{z}} \\
& \theta_{2}=\tan ^{-1} \frac{\left|\mathbf{O}_{\mathbf{S}}-\ddot{\mathbf{r}}_{\mathbf{K S}}\right|_{x}}{\left|\mathbf{O}_{\mathbf{S}}-\ddot{\mathbf{r}}_{\mathbf{K S}}\right|_{z}}
\end{aligned}
$$

Since the knee F-E $\varphi$ is equal to the difference between $\theta_{2}$ and $\theta_{1}$, the following holds

$$
\varphi=\theta_{2}-\theta_{1}
$$

Though the method shown here is similar to that proposed by Dejnabadi et al. (2005), the calculations have been simplified by considering the sensor measurements as the measurements at the center of the link model as shown in Fig.2. 


\subsection{Creating stick figure model}

Stick figure representations of the volunteers were created to be able to visually confirm the positions of the lower limb segments during walking. The left and right hip and knee joint angles, and the segment lengths were used to create a relative coordinate system.

The origin of the relative coordinate system $o(0,0,0)$ was at the median point between the right and left hip joints. The coordinates for RH, LH, RS, LS, RA, and LA were obtained with the following

$$
\begin{aligned}
& \beta H(x, y, z)=\left(0,-\frac{L_{H H}}{2}, 0\right) \\
& \beta K(x, y, z)=\left(\left(\begin{array}{c}
0 \\
0 \\
-\frac{L_{H H}}{2}
\end{array}\right)+d_{\beta} e_{\beta}\left(\begin{array}{c}
0 \\
0 \\
-L_{\text {thigh }}
\end{array}\right)\right)^{\mathbf{T}} \\
& \beta A(x, y, z)=\beta K+\left(d_{\beta} e_{\beta} f_{\beta}\left(\begin{array}{c}
0 \\
0 \\
-L_{\text {shank }}
\end{array}\right)\right)^{\mathbf{T}}
\end{aligned}
$$

Here, $\beta$ is either right or left ( $\mathrm{R}$ or $\mathrm{L}$ ), and $\mathrm{d}$, e, and $\mathrm{f}$ are rotation matrices.

$$
d_{\beta}=\left(\begin{array}{ccc}
\cos \theta_{\beta_{-} p i t c h} & 0 & \sin \theta_{\beta_{-} p i t c h} \\
0 & 1 & 0 \\
-\sin \theta_{\beta_{-} \text {pitch }} & 0 & \cos \theta_{\beta_{-} \text {pitch }}
\end{array}\right)
$$




$$
\begin{aligned}
e_{\beta} & =\left(\begin{array}{ccc}
1 & 0 & 0 \\
0 & \cos \theta_{\beta_{-} \text {roll }} & -\sin \theta_{\beta_{-} \text {roll }} \\
0 & \sin \theta_{\beta_{-} \text {roll }} & \cos \theta_{\beta_{-} \text {roll }}
\end{array}\right) \\
f_{\beta} & =\left(\begin{array}{ccc}
\cos \varphi_{\beta} & 0 & \sin \varphi_{\beta} \\
0 & 1 & 0 \\
-\sin \varphi_{\beta} & 0 & \cos \varphi_{\beta}
\end{array}\right)
\end{aligned}
$$

$L_{H H}$ is the distance between the right and left hip joints, $L_{t h i g h}$ is the distance between the hip and knee joints, $L_{\text {shank }}$ is the distance between the knee and ankle joints, and $\theta_{\beta}$ pitch, $\theta_{\beta \_ \text {roll }}$, and $\varphi_{\beta}$ are the pitch, the roll and the F-E angle of the knee.

To convert the position of the joints in the relative coordinate system into the base coordinate system, the time of heel contact was used. It was reported elsewhere that a sudden drop in the acceleration data can be used to detect heel contact (Currie et al., 1992; Auvinet et al., 2002; Mansfield and Lyons, 2003), and this work used the acceleration at the shank, RS and LS, to determine the time when the leg was set on the ground. Once the point when the leg is set on the ground is determined, the ankle joint, RA or LA, is defined as the $(\mathrm{X}, \mathrm{Y}, 0)$ of the base coordinate system. Since the relative position of the other joint positions, $\mathrm{RK}, \mathrm{LK}, \mathrm{RH}$, and $\mathrm{LH}$, are known these positions in the base coordinate system can be calculated from the ankle joint RA or LA (Fig. 2(c)). 


\section{Experiment}

Three healthy volunteers took part in the experiments and details of the volunteers are shown in Table 2. For the experiments, four sensor units were placed at the lower limb (left and right thigh [LT, RT], left and right shank [LS, RS]) of the volunteers (Fig.3). For comparison, a reference motion analysis system (DIPP-Motion Pro, Ditect Co., Ltd.) was used to track reflective markers on the volunteers as well. The volunteers walked for 5 meters on a flat floor inside the laboratory for three trails. The walking velocity was fixed to a cadence of 88 steps/min using a digital metronome (TU-80, Roland Corporation). Though the proposed system can measure walking for longer distances, the measurement was limited to $5 \mathrm{~m}$ due to the range of the camera system. Measurements for each volunteer were made to obtain the distances between each of the lower limb joints.

To prevent sensor attachment errors, measurements of each sensor unit were made before and after the trials of each volunteer. The measurements of each sensor were taken in two postures, standing upright and sitting flat with outstretched legs on the floor. Using the measurements of the two different postures, a calibration of the three orthogonal axes for the sensor units was conducted. The calibration aligned one 
axis to the longitudinal direction of the segment, one axis to the anterior direction, and one axis to the left lateral direction. 


\section{Results}

Figures 4 and 5 show the results for the measurements of the hip F-E, hip A-A and knee F-E during gait. The vertical axis represents the angles in degrees and the horizontal axis the time in seconds. The thick line represents the joint angles measured for the right leg and the thin line represents the angles of the left leg. The measurements are of the three volunteers (a), (b), and (c).

A comparison of hip F-E, hip A-A, and knee F-E between this method and the camera system is possible by looking at Fig. 6 and 7. The vertical axis represents the angles in degrees and the horizontal axis the percentage of one gait cycle. The joint angles calculated using this method are shown by the thick lines and the reference camera system by the thin lines. The phase lag in the peak flexion joint angle, observed in Fig.7, could be caused by the moving average used to remove noise from the raw acceleration and angular velocity data. Table 3 shows the RMSE, absolute deviation (AD) of error, correlation coefficient (CC) and percentage of variance unexplained (PVU) between the joint angles calculated from this method and that of camera for all three volunteers.

Figure 8 shows the hip and knee joint trajectories in the horizontal plane. The vertical and horizontal axes represent the measurements in $\mathrm{x}$ and $\mathrm{y}$ coordinates 
respectively, with $(0,0)$ as the center of the right and left hip joint.

Figure 9 is a stick figure representation of a volunteer using this method and the reference camera system. Here the abdomen segment for the stick figure is shown for illustration purposes only. Software developed for this work showed the volunteer's gait in the $\mathrm{X}-\mathrm{Z}$ plane and in the $\mathrm{Y}-\mathrm{Z}$ plane. 


\section{Discussion}

First, the average of the hip F-E for all the volunteers was: RSME $=8.72$ deg, $\mathrm{AD}=6.57$ deg, $\mathrm{CC}=0.88$ and $\mathrm{PVU}=20.05 \%$. With the $\mathrm{CC}$ and the PVU low, the results show that this method measures the hip F-E with high consistency to that of the camera. For the hip A-A the average values were: $\mathrm{RSME}=4.96 \mathrm{deg}, \mathrm{AD}=3.30 \mathrm{deg}$, $\mathrm{CC}=0.72$ and $\mathrm{PVU}=39.29 \%$. The low RSME and AD are caused by the absolute range of the A-A motions being smaller than to F-E motions of the hip. The low CC and high PVU may indicate an effect of the internal-external (I-E) rotation during gait motion. The method here did not consider I-E motion, and including measurements of this could improve the results, an issue to consider in future work. The average knee F-E values were: $\mathrm{RSME}=6.79 \mathrm{deg}, \mathrm{AD}=4.65 \mathrm{deg}, \mathrm{CC}=0.92$ and $\mathrm{PVU}=14.60 \%$. The knee F-E had higher CC and lower PVU averages than the hip F-E. The CC average of 0.92 for the knee F-E was consistent with the results using wearable sensors provided by Tong and Granat (1999), and Dejnabadi et al. (2005) where the CC were 0.93 and 0.99 respectively. It may be concluded that the knee F-E can be measured with significant accuracy using wearable sensors.

The knee trajectories in Fig. 8 showed that the knee and ankle joint trajectories were symmetric for both the right and left legs, with the exception of one volunteer (c). 
It was not established why the ankle joint trajectories were so different for the right leg, it is suspected that the sensor $\mathrm{s}^{\mathrm{RS}}$ could have moved during the trial. This would have caused errors in the orientation calculations for the right shank.

The method presented here showed a strong correlation with the camera system data and involved significantly less calculation than reported in previous reports (Takeda et al., 2009). Further, the method here does not require measurements of the cyclic gait over long periods of time. One limitation of this work is in the assumption of constant velocity in the walking direction, and the method introduced here is based on the assumption that the hip joint movement includes only centripetal and tangential acceleration. It has been reported that the anterior-posterior acceleration of the trunk segment increased with walking velocity (Zijlstra and Hof, 2003), and as the current work conducted experiments at fairly low velocity (88 steps/min), the effect of any anterior-posterior acceleration may not have been apparent. However, non-constant motion or gaits at higher velocities may lead to measurement errors and this has to be controlled for. In addition, errors introduced by the attachment of the sensors is an issue with any kind of wearable sensor. During movement the attached sensor may move causing errors in the measurements. This problem can be controlled for by conducting a predefined motion calibration process before each trial. The work here used the upright 
and sitting positions to align the sensor axes in the sagittal plane. Future work will be needed to develop a more secure method for fixation of the sensors.

With the limitations detailed here, the work here shows that wearable acceleration and gyro sensors can provide quantitive measurements of human gait motion with high accuracy as expressed by joint angles, joint trajectories and presented in stick figures. Future work will be required to develop a method for calculating the internal-external rotation of the hip joints to provide more accurate results. 


\section{Awknowlegement}

The authors wish to express thanks to H. Miyagawa of the Laboratory of Biomechanical Design (Division of Human Mechanical Systems and Design, Hokkaido University), for support and cooperation in the experiments and computer data analysis of this study.

\section{Conflict of interest's statement}

There are no actual or potential conflicts of interest related to the research reported here.

\section{References}

Auvinet, B., Gloria, E., Renault, G., Barrey, E., 2002. Runner's stride analysis: comparison of kinematic and kinetic analyses under field conditions. Science \& Sports, 17, 92-94.

Bouten, C.V.C., Koekkoek, K.T.M., Verduin, M., Kodde, R., Janssen, J.D., 1997. A triaxial accelerometer and portable data processing unit for the assessment of daily physical activity. IEEE Transactions on Biomedical Engineering, 44, 136-147.

Brodie, M.A., Walmsely, A., Page, W., 2008. The static accuracy and calibration of inertial measurement units for 3D orientation. Computer Methods in Biomechanics and Biomedical Engineering, 11, 641-648. 
Bussmann, J. B. J., van de Laar, Y. M., Neeleman, M. P., Stam, H. J., 1998. Ambulatory accelerometry to quantify motor behaviour in patients after failed back surgery: a validation study. Pain, 74, 153-161.

Currie, G., Rafferty, D., Duncan, G., Bell, F., \& Evans, A. L., 1992. Measurement of gait by accelerometer and walkway: a comparison study. Medical and Biological Engineering and Computing, 30, 669-670.

Davis, R. B. III, Ounpuu, S., Tyburski, D., Gage, J. R., 1991. A gait analysis data collection and reduction technique. Human Movement Science, 10, 575-587.

Dejnabadi, H., Jolles, B.M., Aminian, K., 2005. A new approach to accurate measurement of uniaxial joint angles based on a combination of accelerometers and gyroscopes. IEEE Transactions on Biomedical Engineering, 52, 1478-1484.

Findlow, A., Goulermas, J.Y., Nester, C., Howard, D., Kenny, L.P.J., 2008. Predicting lower limb joint kinematics using wearable motion sensors. Gait

Foerster, F., Smeja, M., Fahrenberg, J., 1999. Detection of posture and motion by accelerometry: a validation study in ambulatory monitoring. Computers in Human Behavior, 15, 571-583.

Luinge, H. J. and Veltink, P. H., 2005. Measuring orientation of human body segments using minature gyroscopes and accelerometers. Medical \& Biological Engineering \& 
Computing, 43, 273-282.

Morris, J.R.W., 1973. Accelerometry - A technique for the measurement of human body movements. Journal of Biomechanics, 6, 729-736.

Mansfield, A., \& Lyons, G. M. (2003). The use of accelerometry to detect heel contact events for use as a sensor in FES assisted walking, Medical Engineering \& Physics, 25, 879-885.

Roetenberg, D.; Slycke, P. J.; Veltink, P. H, 2007. Ambulatory position and orientation tracking fusing magnetic and inertial sensing. IEEE Transactions on Biomedical Engineering, 54, 883-890.

Takeda, R., Tadano, S., Todoh, M., Morikawa, M., Nakayasu, M., Yoshinari, S., 2009. Gait analysis using gravitational acceleration measured by wearable sensors. Journal of Biomechanics, 43, 223-233.

Tong, K. and Granat, M. H., 1999. A practical gait analysis system using gyroscopes. Medical Engineering and Physics, 21, 87-94.

Veltink, P.H., Bussmann, HansB.J., de Vries, W., Martens, WimL.J., Van Lummel, R.C., 1996. Detection of static and dynamic activities using uniaxial accelerometers. IEEE Transactions on Rehabilitation Engineering, 4, 375-385.

Zhu, R. and Zhou, Z., 2004. A real-time articulated human motion tracking using 
tri-axis inertial/magnetic sensors package. IEEE Transactions on Neural Systems and Rehabilitation Engineering, 12, 295-302.

Zijlstra, L. and Hof, A. L., 2003. Assessment of spatio-temporal gait parameters from trunk accelerations during human walking. Gait and Posture, 18, 1-10. 


\section{Figure Legends}

Figure 1 Gait model and coordinate systems. The $X, Y, Z$ coordinates represents the base coordinate system, where the $X$ axis is the walking direction, the $Y$ axis is the left-lateral direction, and the $Z$ axis is the direction opposite to gravity. The sensors are placed on 4 locations RT, LT, RS and LS.

Figure 2 Measurements for hip and knee joint angles of the right leg. (a) $r_{H T}$ is the distance from $\mathrm{RH}$ to $\mathrm{S}^{\mathrm{RT}}$, $\mathrm{r}_{\mathrm{KT}}$ is the distance from $\mathrm{RK}$ to $\mathrm{S}^{\mathrm{RT}}$, $\mathrm{r}_{\mathrm{KS}}$ is the distance from $\mathrm{RK}$ to $\mathrm{S}^{\mathrm{RS}}$. (b1) $\theta_{1}$ is the inclination angle of $\mathbf{a}_{\mathbf{x}}-\mathbf{g}$ against RH-RK, $\theta_{2}$ is the inclination angle of $\mathbf{a}_{\mathbf{x}}-\mathbf{g}$ against RK-RA. (b2) the difference of $\theta_{2}$ and $\theta_{1}$ is equivalent to the knee joint flexion angle.

Figure 3 Conversion of relative coordinate joint positions to base coordinate system. Heel contact is used to determine which foot is set on the ground. The $\mathrm{Z}$ coordinate of the ankle joint set on the ground is considered to be 0 .

Figure 4 Sensor attachment locations during experiment. Reflective markers are placed on the volunteers to track movements using reference camera motion analysis system. 
Figure 5 Hip joint flexion-extension and abduction-adduction for subjects (a), (b) and (c). are shown. Vertical axis represents joint angles in degrees. $0^{\circ}$ is the joint angle during stance, negative values represent flexion or abduction and positive values represent extension or adduction. Horizontal axis represents time in seconds. The dark line is the joint angles for the right and light line is the joint angle for the left.

Figure 6 Knee joint flexion-extension for subjects (a), (b) and (c). are shown. Vertical axis represents joint angles in degrees. $0^{\circ}$ is the joint angle during stance, negative values represent flexion and positive values represent extension. Horizontal axis represents time in seconds. The dark line is the joint angles for the right and light line is the joint angle for the left.

Figure 7 Hip joint angle comparisons between this method and camera motion analysis for subjects (a), (b) and (c). Vertical axis represents joint angles in degrees and horizontal axis represents the percentage in one gait cycle. The dark lines are the joint angles calculated using this method and the light lines are those of camera motion analysis. 
Figure 8 Knee joint angle comparisons between this method and camera motion analysis for subjects (a), (b) and (c). Vertical axis represents joint angles in degrees and horizontal axis represents the percentage in one gait cycle. The dark lines are the joint angles calculated using this method and the light lines are those of camera motion analysis.

Figure 9 Knee and ankle joint trajectories calculated using this method for subjects (a), (b) and (c). The dark thin line is the trajectory of the left and the light line is that of right leg. The trajectories are represented in the $x-y$ plane of relative coordinate system o. Both vertical and horizontal axes are measurements in $\mathrm{cm}$.

Figure 10 Stick figure visualization program. The program shows the stick figure representations of this method (top) and camera (bottom). This program shows the both walking in the $\mathrm{X}-\mathrm{Z}$ base coordinate plane (left) and Y-Z base coordinate plane (right) simultaneously. The dark heavy line is 0 in the $\mathrm{Z}$ coordinate and shown as reference. 


\section{Table Legend}

Table 1 The RSME and coefficient correlation of the hip flexion-extension, hip abduction-adduction and knee flexion-extension. The results are shown separately for subjects (a), (b) and (c). 
Figure 1

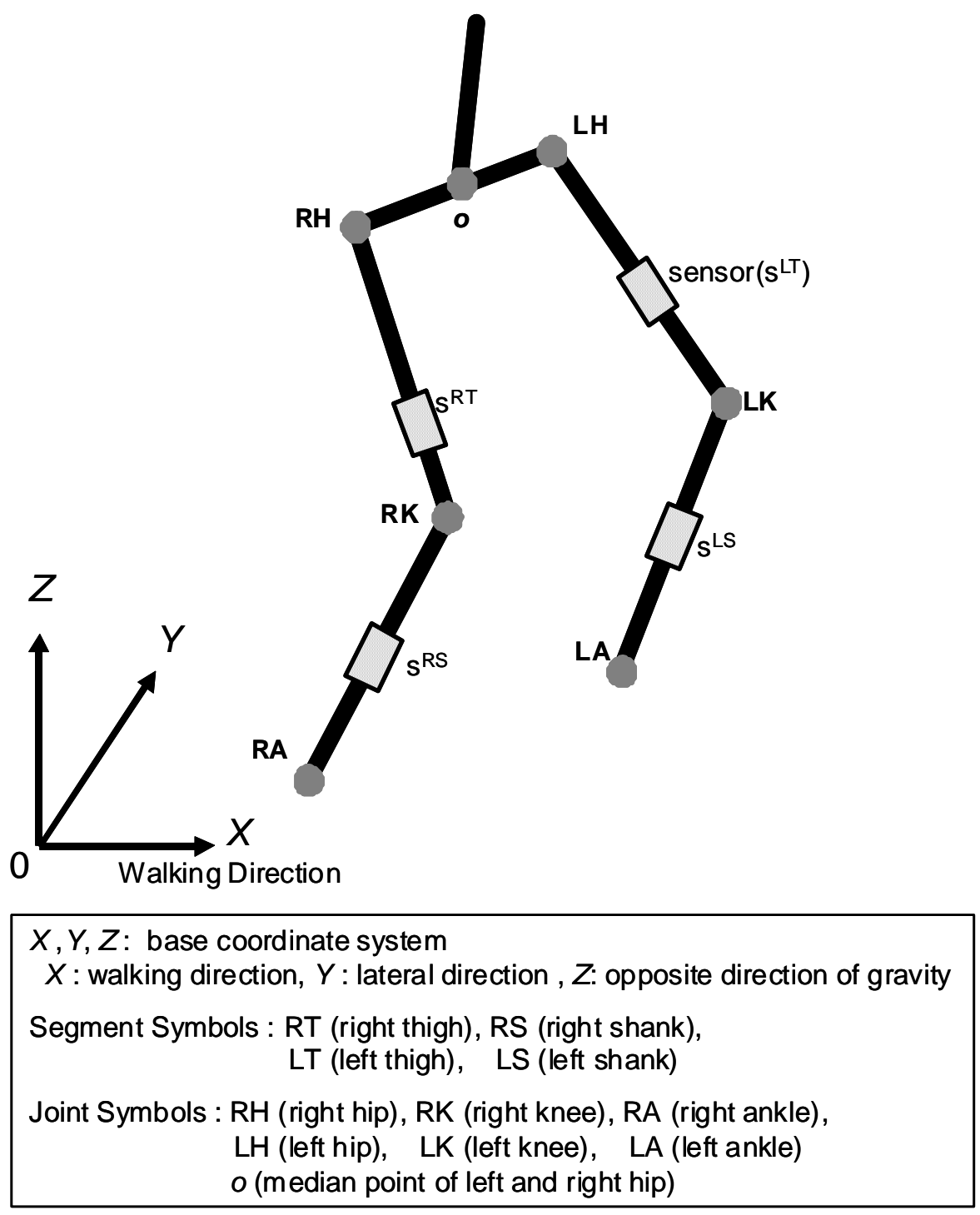


Figure 2

(a)

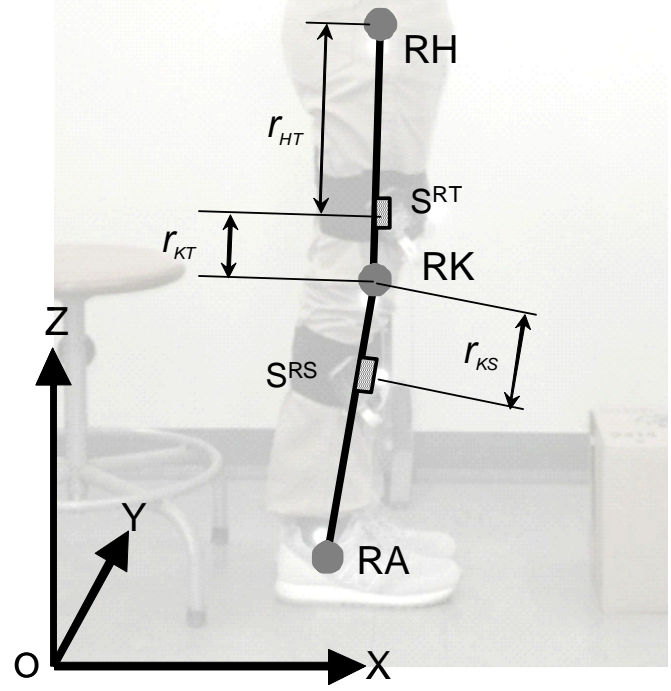

(b1)

(b2)
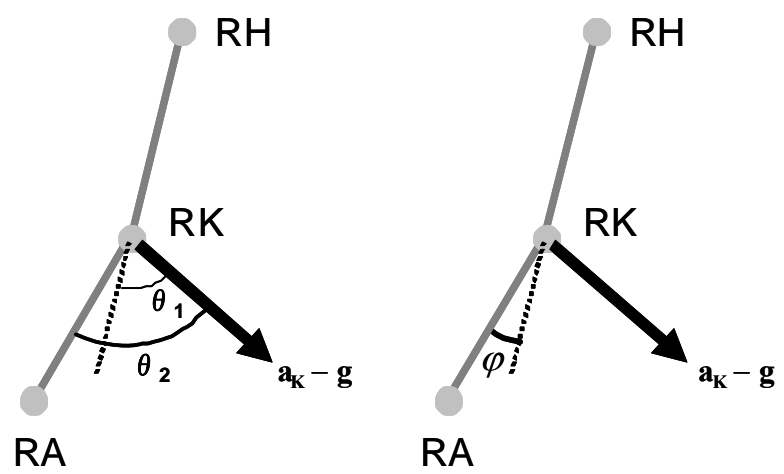
Figure 3

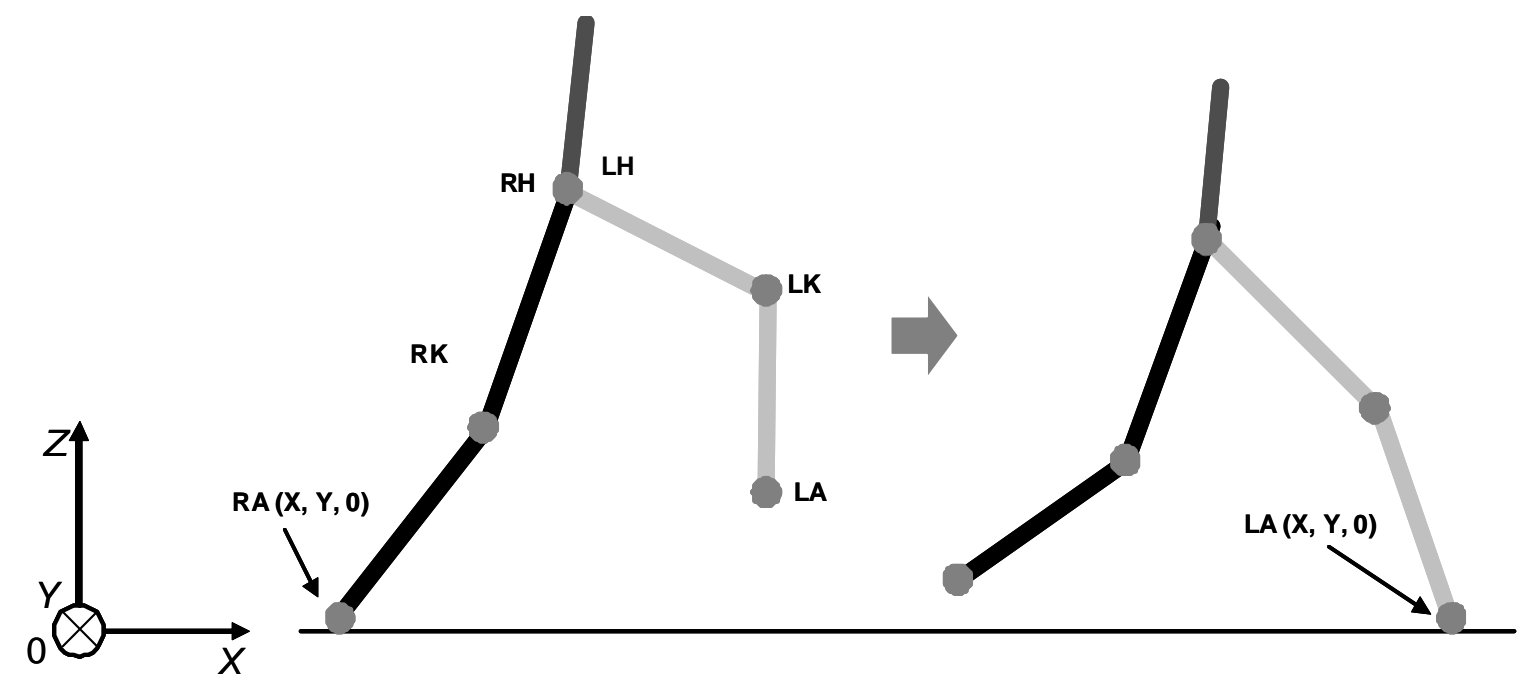


Figure 4

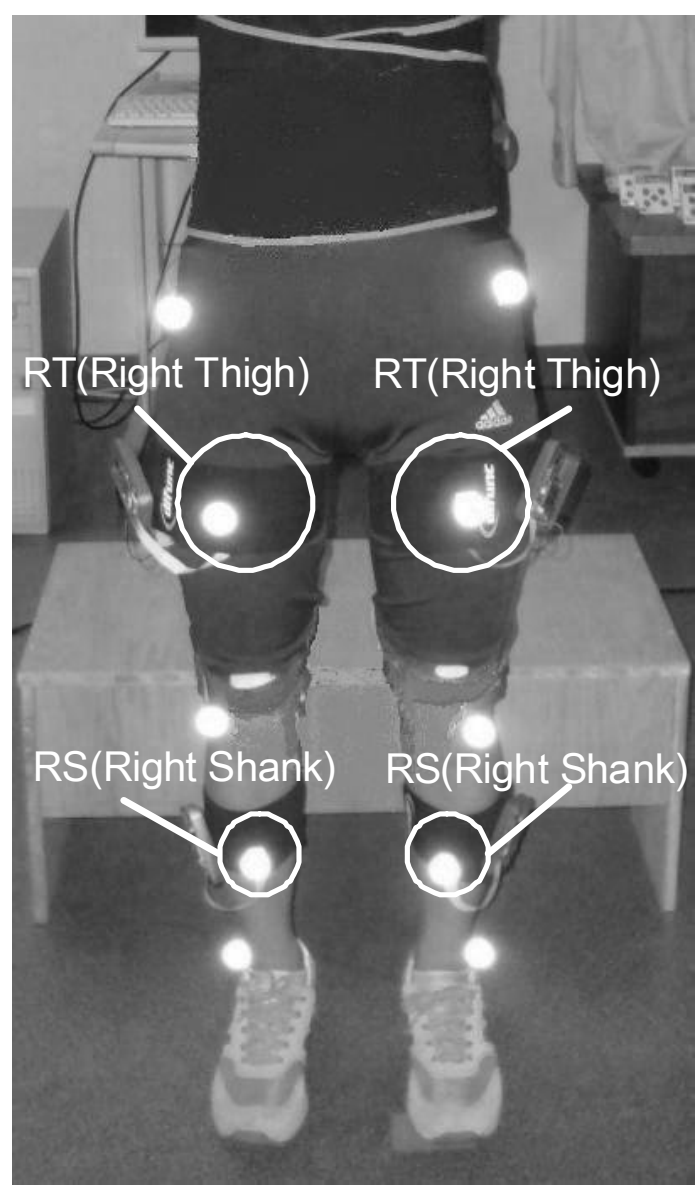


Figure 5
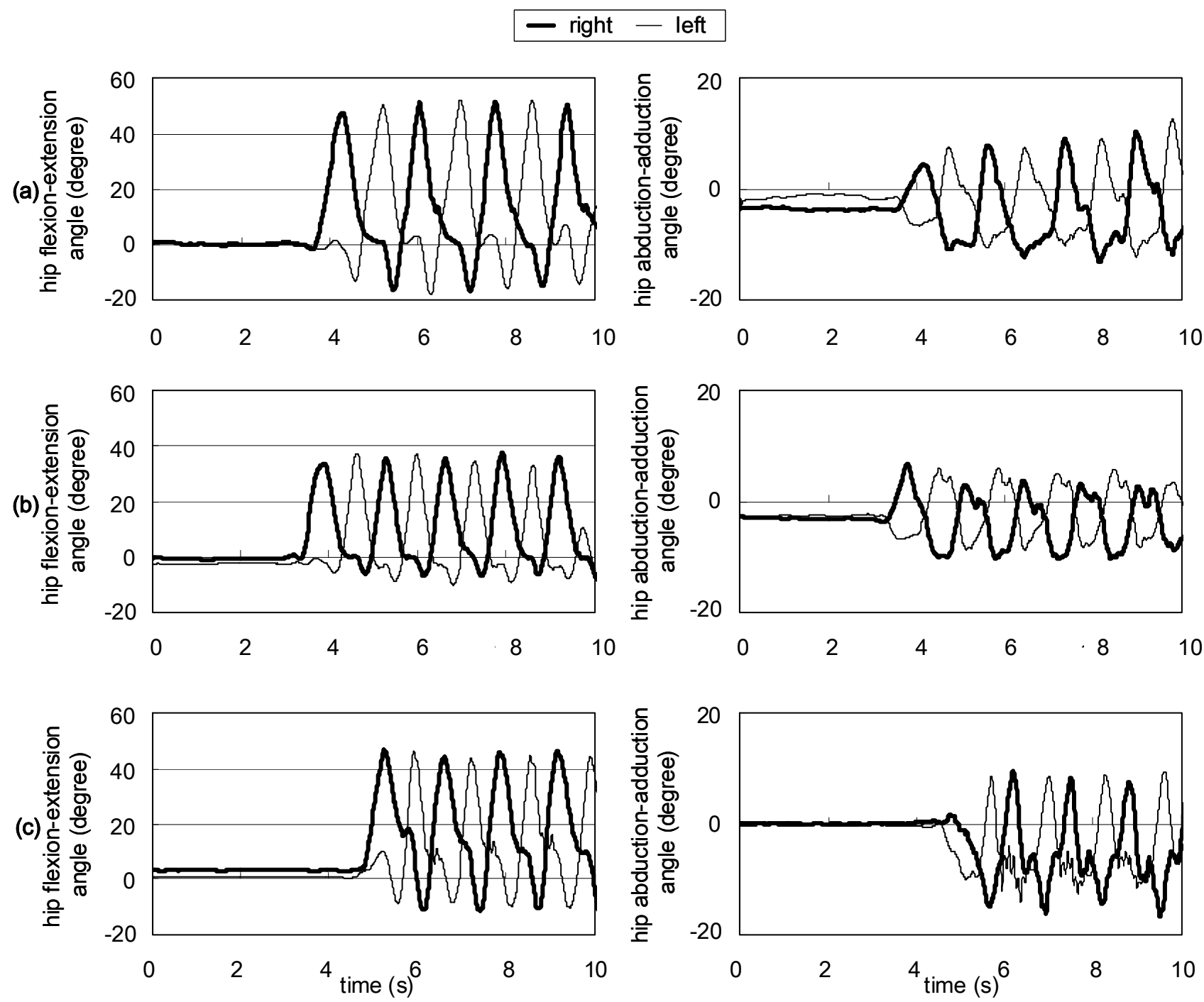
Figure 6
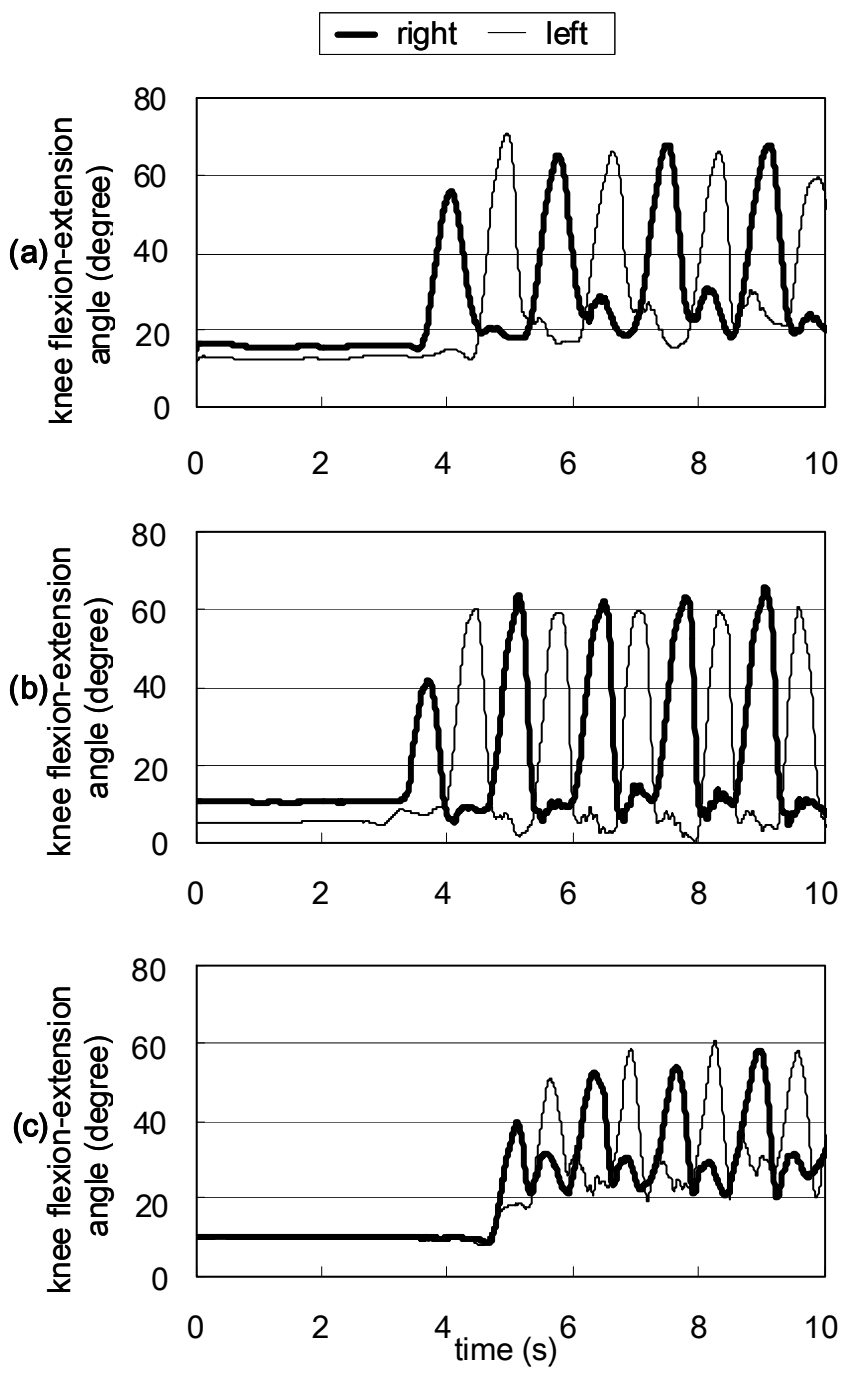
Figure 7
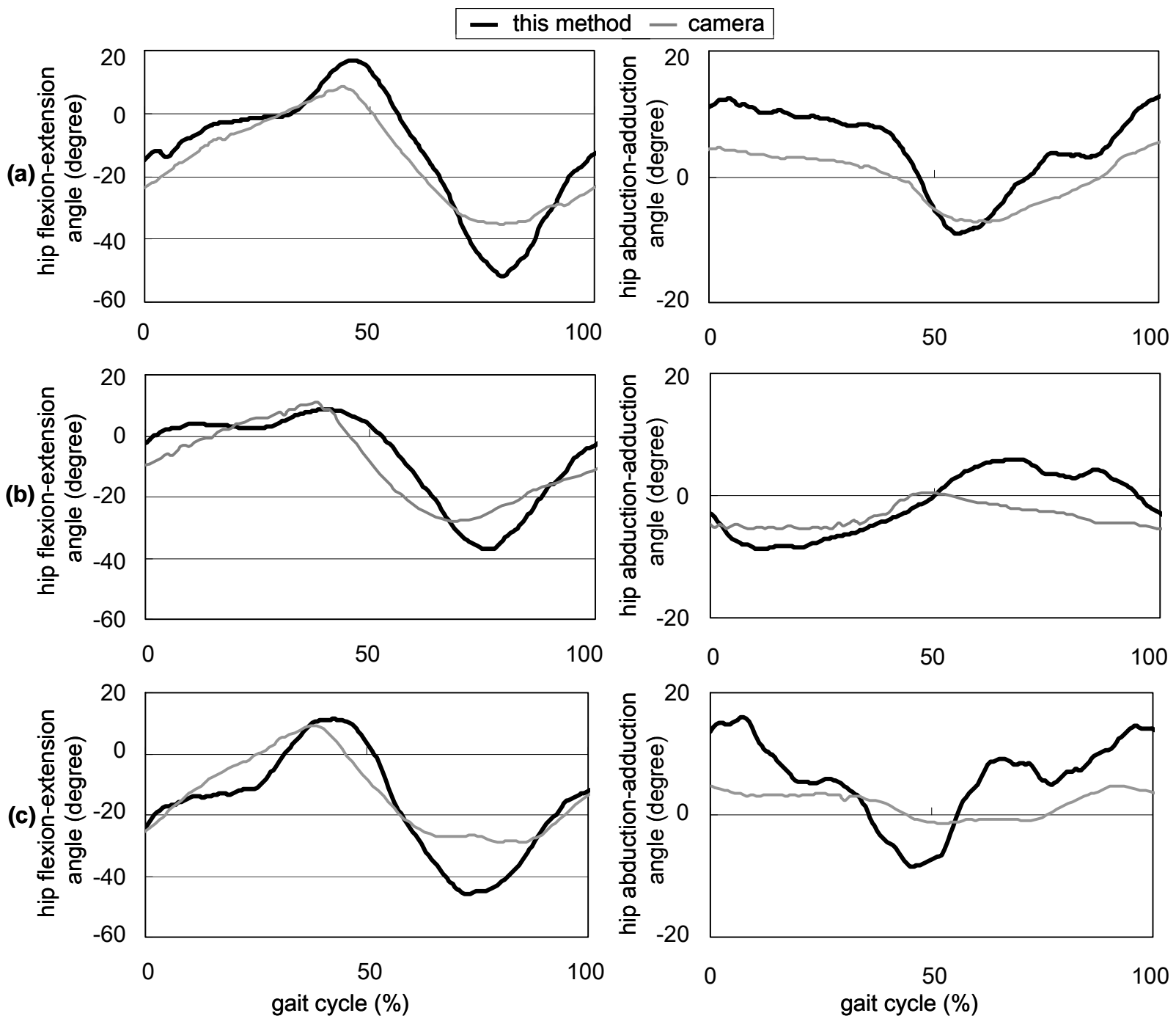
Figure 8
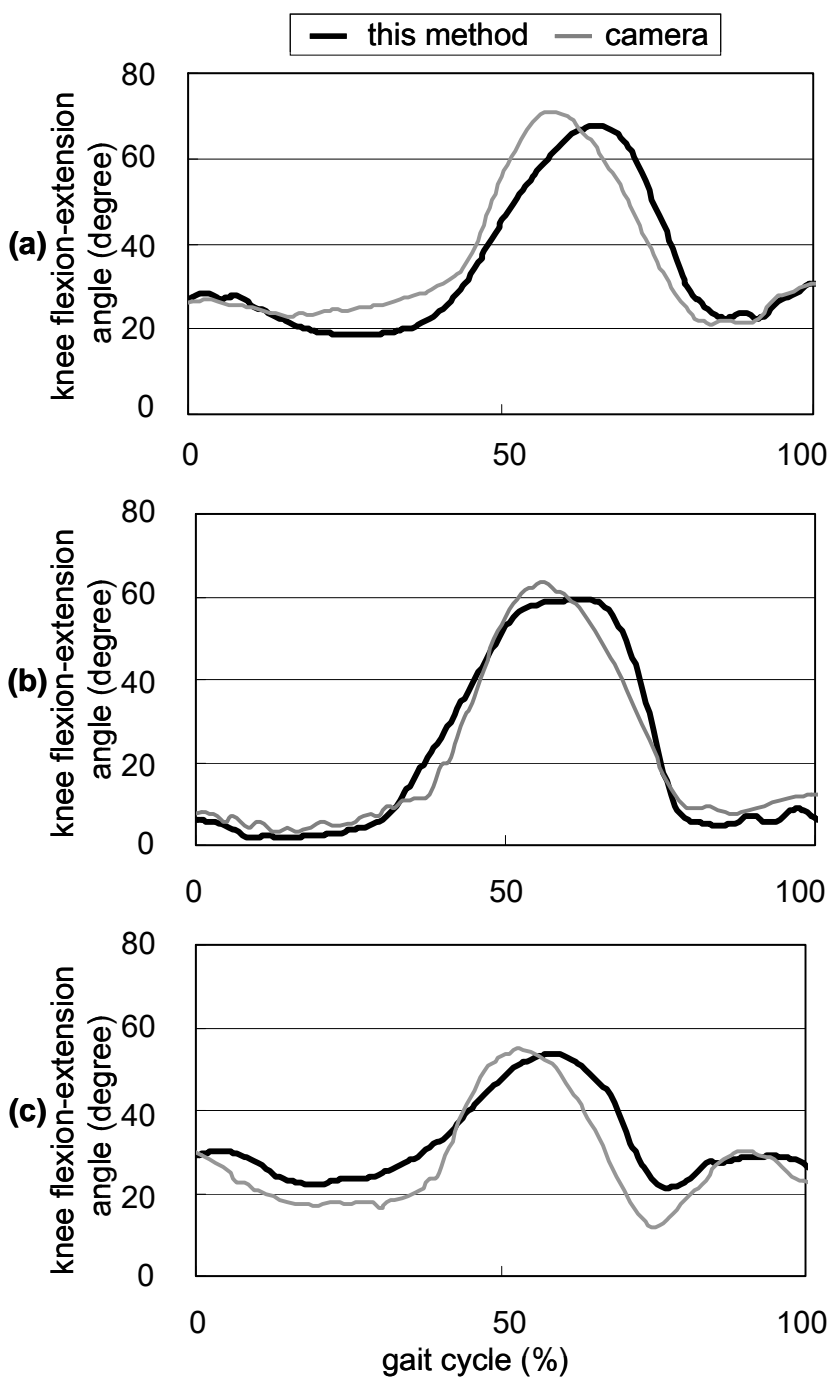
Figure 9
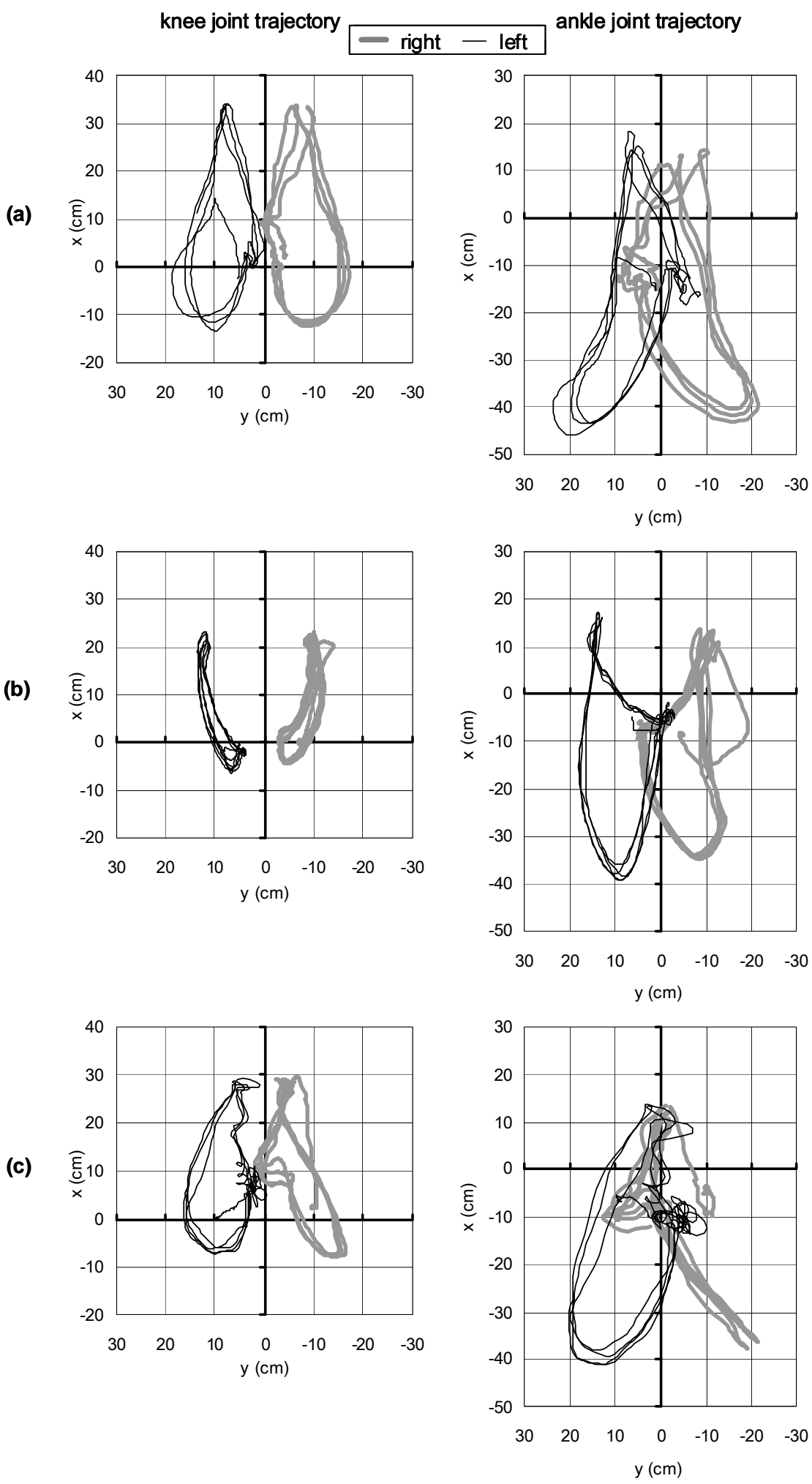
Figure 10

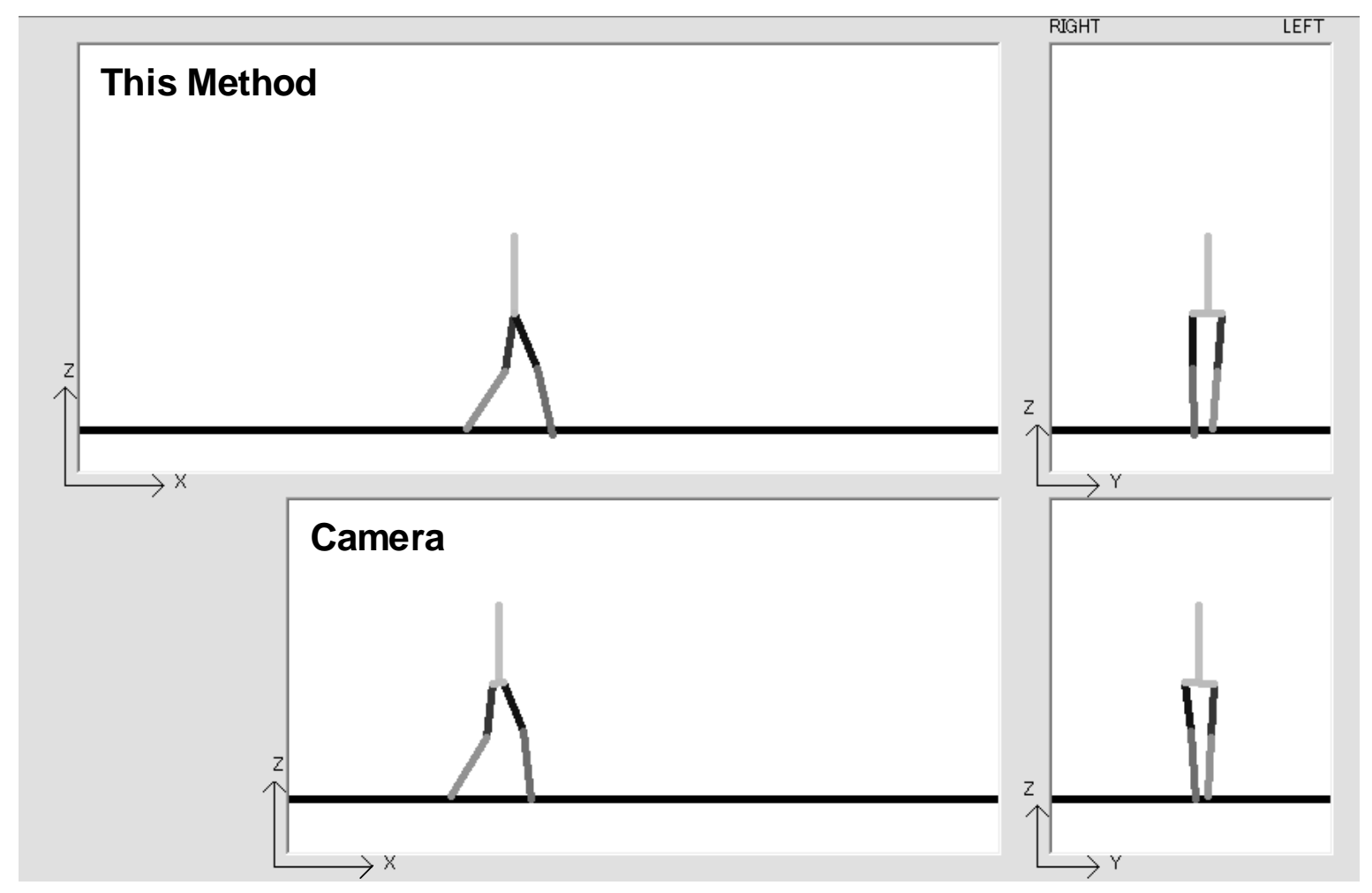


Table 1

\begin{tabular}{lcccccc}
\hline & $\begin{array}{c}\text { Hip Angle } \\
\text { (Flexion- } \\
\text { Extension) } \\
\text { RMSE }\end{array}$ & $\begin{array}{c}\text { Hip Angle } \\
\text { (Abduction- } \\
\text { Adduction) }\end{array}$ & \multicolumn{2}{c}{$\begin{array}{c}\text { Knee Angle } \\
\text { (Flexion- } \\
\text { Extention) }\end{array}$} \\
\hline Subject (a) & 10.34 & 0.918 & 5.24 & 0.892 & 6.69 & 0.928 \\
RMSE & CC & $\begin{array}{c}\text { RMSE } \\
\text { (deg) }\end{array}$ & CC \\
Subject (b) & 6.45 & 0.902 & 4.10 & 0.628 & 6.58 & 0.954 \\
Subject (c) & 9.38 & 0.845 & 5.55 & 0.644 & 7.10 & 0.892 \\
\hline
\end{tabular}

